\title{
Nonequilibrium Dynamics and Aging in the Three-Dimensional Ising Spin Glass Model
}

\author{
Heiko Rieger* \\ Physics Department, University of California, Santa Cruz, CA 95064, USA \\ HLRZ c/o KFA Jülich, Postfach 1913, 5170 Jülich, Germany
}

\begin{abstract}
The low temperature dynamics of the three dimensional Ising spin glass in zero field with a discrete bond distribution is investigated via MC simulations. The thermoremanent magnetization is found to decay algebraically and the temperature dependent exponents agree very well with the experimentally determined values. The nonequilibrium autocorrelation function $C\left(t, t_{w}\right)$ shows a crossover at the waiting (or aging) time $t_{w}$ from algebraic quasi-equilibrium decay for times $t \ll t_{w}$ to another, faster algebraic decay for $t \gg t_{w}$ with an exponent similar to one for the remanent magnetization.
\end{abstract}

PACS numbers: 75.10N, 75.50L, 75.40G.

*Present address: Institut für Theoretische Physik, Universität zu Köln, 5000 Köln 41, Germany. 
The measurement of dynamical nonequilibrium quantities in real spin glasses [1] has a long history. The typical experiment that has been conducted many times [2, 3] is the following: Within a magnetic field the spin glass (for instance $\mathrm{Cu}(\mathrm{Mn}), \mathrm{Au}(\mathrm{Fe}), \mathrm{Fe}_{0.5} \mathrm{Mn}_{0.5} \mathrm{TiO}_{3}$, $\left(\mathrm{Fe}_{x} \mathrm{Ni}_{(1-x)}\right)_{75} \mathrm{P}_{16} \mathrm{~B}_{6} \mathrm{Al}_{3}, \mathrm{Cd}_{x} \mathrm{Mn}_{(1-x)} \mathrm{Te}$, etc.) is cooled down to temperatures below the freezing temperature $T_{g}$ and either immediately or after a certain waiting time $t_{w}$ the field is switched off. Then the so called (thermo)remanent magnetization $M_{\mathrm{rem}}(t)$ is measured as a function of time $t$. The asymptotic time dependence of this quantity is found to be algebraic well below $T_{g}\left(T / T_{g} \leq 0.98\right)$ in the short range Ising spin glass $\mathrm{Fe}_{0.5} \mathrm{Mn}_{0.5} \mathrm{TiO}_{3}$ 近.5 and in an amorphous metallic spin glass $\left(\mathrm{Fe}_{x} \mathrm{Ni}_{(1-x)}\right)_{75} \mathrm{P}_{16} \mathrm{~B}_{6} \mathrm{Al}_{3}[6]$. Furthermore the timedependence of the remanent magnetization depends on the waiting time $t_{w}$, a phenomenon called aging [2].

Several attempts have been made to explain this behavior theoretically [0,8,9, 10, 11] and a wide variety of functional forms for the time dependence of the remanent magnetization is found. The problem lays in the fact that starting from a microscopic model or modelHamiltonian one encounters insurmountable difficulties in trying to solve the nonequilibrium dynamics. Therefore additional assumptions have to be made and the final outcome stretched exponential [7], algebraic [10,[1] or logarithmic [9] decay - depends on them. Even within the mean field approximation it is hard to obtain any analytical [12,13] or semianalytical [14] results.

Once a microscopic model for a spin glass has been formulated, one can in principle try to extract its macroscopic behavior via Monte Carlo (MC) simulations. In contrast to an analytical treatment, where the calculation of dynamical nonequilibrium quantities within the spin glass phase (instead of those characterizing equilibrium, see [15]) is even more complicated, MC simulation can be done with less effort for certain nonequilibrium situations, since equilibration times reach astronomical values in case of spin glasses [16, 17, 18]. The remanent magnetization with zero waiting time has been investigated numerically for the mean field version of a spin glass model [9, [19] and for the three dimensional EA (Edwards- 
Anderson) model only right at the critical temperature [20]. Quite recently attempts have been made to investigate the whole temperature range below $T_{g}$ numerically [21,22]. However, a systematic MC study of nonequilibrium correlations and aging phenomena within the frozen phase $\left(T<T_{c}\right)$ of the three-dimensional EA spin glass model has not been made up to now. The results such an investigation, its theoretical implications and comparison with experiments will be reported in this letter.

The system under consideration is the three-dimensional Ising spin-glass with nearest neighbor interactions and a discrete bond distribution. Its Hamiltonian is

$$
\mathcal{H}=-\sum_{\langle i j\rangle} J_{i j} \sigma_{i} \sigma_{j},
$$

where the spins $\sigma_{i}= \pm 1$ occupy the sites of a $L \times L \times L$ simple cubic lattice with periodic boundary conditions and the random nearest neighbor interactions $J_{i j}$ take on the values +1 or -1 with probability $1 / 2$. We consider single spin flip dynamics and used a special, very fast implementation of the Metropolis algorithm on a Cray YM-P (see ref. [23] for details). The simulations were done in the frozen phase, that means at temperatures below $T_{c}=1.175 \pm 0.025$ (see ref. [16, 17, 18]). All measured quantities are averaged over at least 128 samples (smaller system sizes were averaged over up to 1280 samples). The system size was increased until no further size dependence of the results has been observed within the simulation time $\left(t \leq 10^{6}\right)$, which is measured in MC sweeps through the whole lattice. It turns out that $L=32$ is large enough for this time range (cf. [18]).

The system was prepared in a fully magnetized initial configuration and then the simulation was run for a time $t_{w}$ (=waiting time) and then the spin configuration $\underline{\sigma}\left(t_{w}\right)$ was stored. From now on after each MC step (data were then averaged over appropriate time intervals, cf. [18]) the following correlation function was measured:

$$
C\left(t, t_{w}\right)=\frac{1}{N} \sum_{i} \overline{\left\langle\sigma_{i}\left(t+t_{w}\right) \sigma_{i}\left(t_{w}\right)\right\rangle},
$$

where $\langle\cdots\rangle$ means a thermal average (i.e. an average over different realizations of the thermal noise, but the same initial configuration) and the bar means an average over different 
realizations of the bond-disorder. The quantity $C(t, 0)$ corresponds to the remaining magnetization of the system after a time $t$.

$$
M_{\text {rem }}(t)=C(t, 0) .
$$

This quantity is directly related to the experimentally determined thermoremanent magnetization with zero waiting time (i.e. without aging) and nearly saturated initial magnetization. The result for the remanent magnetization $M_{\text {rem }}(t)$ is shown in fig. [ ] within a log-log plot. Its decay clearly obeys a power law for large times and temperatures in the range $1.1 \geq T \geq 0.5$. The exponent $\lambda(T)$ for the fit

$$
M_{\mathrm{rem}}(t) \propto t^{-\lambda(T)}, \quad\left(t \geq 10^{2}\right)
$$

is plotted in fig. 2, upper curve. It starts at $\lambda=0.36 \pm 0.01$ for $T=1.1$ (and can be extrapolated via the fit indicated in figure 2 to $0.39 \pm 0.01$ for $\mathrm{T}=\mathrm{T}_{c}$, which was already found in [20]) and decreases monotonically with temperature. For the short range Ising spin glass $\mathrm{Fe}_{0.5} \mathrm{Mn}_{0.5} \mathrm{TiO}_{3}$ and for certain amorphous metallic spin glasses not only the same algebraic decay of the remanent magnetization has been observed, but also the shape of the functional temperature dependence of the exponent $\lambda(T)$ and even its numerical values are in excellent agreement: from figure $4 \mathrm{~b}$ in ref. [6] one may for instance read off $\lambda\left(T_{g}\right) \approx 0.38$ and $\lambda\left(0.5 T_{g}\right) \approx 0.12$, concurring within the errorbars to the corresponding data plotted in fig. 2 .

It was argued [9] that the decay of $M_{\text {rem }}(t)$ should be logarithmic (i.e. $M_{\text {rem }}(t) \propto$ $(\ln t)^{-\lambda / \psi}$ ) below $T_{c}$, but a fit of the data in fig. 1 for $T \geq 0.5$ does not yield acceptable results over the range of the observation time. We want to focus some attention to the $T=0.4$ curve: It bends upward in the $\log -\log$ plot for $t>10^{4}$, which could indicate the onset of a slowlier than algebraic decay for temperatures smaller than 0.5 , logarithmic for instance.

Another indication that something new happens at lower temperatures can be obtained by looking at the short time behavior of $M_{\text {rem }}(t):$ At $T \approx 0.5$ a plateau begins to develop 
for $t<10^{2}$, which can clearly be seen for $T=0.4$ and becomes even more pronounced and wider for even smaller temperatures. It can be excactly reproduced in shape and location for smaller and larger sizes and number of samples, which means that it is a physical effect and not only a fluctuation. A possible interpretation might be that the system gets trapped in metastable states, whose lifetime grows with decreasing temperatures.

Next we turn our attention to the correlation function $C\left(t, t_{w}\right)$ with $t_{w}=10^{a}(a=$ $1, \ldots, 5)$. In contrast to $M_{\text {rem }}(t)$ the correlation function $C\left(t, t_{w}\right)$ for $t_{w} \neq 0$ is not directly related to the thermoremanent magnetization $M\left(t, t_{w}\right)$ at time $t+t_{w}$ in a temperature-quench experiment, where the field $H$ is switched off at time $t_{w}$ after the quench (see e.g. 24]). In equilibrium $\left(t_{w} \rightarrow \infty\right) C(t, \infty)$ is related to the relaxation function $R(t, \infty)=M(t, \infty) / H$ via the fluctuation dissipation theorem (FDT) $R(t, \infty)=C(t, \infty) / k_{B} T$. However, in a nonequilibrium situation, like the one considered here, slight differences between them exist 21,25] (e.g. in the location of the maximum relaxation rate). The magnetization that is induced by a small external field $(H \ll 1)$ for model $(1)$ is rather small, therefore the functional form of $M\left(t, t_{w}\right)$ is harder to determine accurately via $\mathrm{MC}$-simulations. This is the reason why in this letter the focus is on $C\left(t, t_{w}\right)$.

A typical set of data for a particular temperature $(T=0.8)$ is shown in fig. 3 in a $\log -\log$ plot. One observes a crossover from a slow algebraic decay for $t \ll t_{w}$ to a faster algebraic decay for $t \gg t_{w}$. The crossover time is simply defined as the intersection of the two straight line fits for short- and long-time behavior in the log-log plot. For the long-time behavior the fit to

$$
C\left(t, t_{w}\right) \propto t^{-\lambda\left(T, t_{w}\right)}, \quad t \gg t_{w}
$$

yields a set of exponents that is depicted in fig. 2. For increasing $t_{w}$ the exponent $\lambda\left(T, t_{w}\right)$ decreases only slightly and the waiting time dependence becomes weaker for lower temperatures. By looking at fig. 3 one observes that it is difficult to extract $\lambda\left(T, t_{w}\right)$ for $t_{w}=10^{4}$ and $10^{5}$ since there are only 2 or 1 decades left to fit the exponent - therefore they are not shown in fig. 2. The exponent describing the short time $\left(t \ll t_{w}\right)$ behavior of $C\left(t, t_{w}\right)$, 


$$
C\left(t, t_{w}\right) \propto t^{-x(T)}, \quad t \ll t_{w},
$$

which is depicted in fig. 团, is independent of the waiting time $t_{w}$. Since the system was able to equilibrate over a time $t_{w}$, all processes occuring on timescales smaller than $t_{w}$ have the charcteristics of equilibrium dynamics and therefore the exponent $x(T)$ is identical to that describing the decay of the equilibrium autocorrelation function $q(t)=\lim _{t_{w} \rightarrow \infty} C\left(t, t_{w}\right)$. The latter was investigated in [18 and the exponents that are reported there for $T \geq 0.7 T_{c}$ agree with the values shown in fig. 团. They also agree with those determined experimentally [5] in the short range Ising spin glass $\mathrm{Fe}_{0.5} \mathrm{Mn}_{0.5} \mathrm{TiO}_{3}$ via the above mentioned relaxation function $R\left(t, t_{w}\right)=M\left(t, t_{w}\right) / H$ for $t \ll t_{w}$ (note that in this quasiequilibrium-regime $C\left(t, t_{w}\right)$ and $R\left(t, t_{w}\right)$ are related via the FDT [21.25], yielding the same exponents for both): close to $T_{g}\left(T / T_{g}=1.029\right)$ they obtain $x=0.07$. Furthermore there seems to be a temperature at about 0.3 , where $x(T)$ becomes zero, which could be another indication for the above mentioned onset of a logarithmic decay of the correlation functions [26].

Although the decay of the nonequilibrium correlations in the temperature range $0.5 \leq$ $T \leq 1.1$ is algebraic rather than logarithmic as predicted by the droplet picture proposed in [9], this picture might not be inappropriate: Let us assume the following scaling law for the dependence of the free energy barriers $B$ on a length scale $L$ of the regions to be relaxed: $B \propto \Lambda \ln L$ instead of $B \propto L^{\psi}$ as in [9]. Then one ends up with an algebraic decay of e.g. the remanent magnetization by observing (see [9]) that the typical length scale of domains $R_{t}$ now grows with time according to $\Lambda \ln R_{t} \sim T \ln t$, which means $R_{t} \propto t^{T / \Lambda(T)}$, leading to equations (3) 6).

In the context of the phenomenological model for the dynamics and aging in disordered systems developped in ref. [11], the algebraic decay of correlations found so far implies that the probability distribution of free energy barriers is exponential in the temperature range of $0.5 \leq T \leq 1.1$ for the system under consideration. Furthermore we would like to mention that a fit to the functional form for the short time behavior $\left(t \ll t_{w}\right) C\left(t, t_{w}\right) \sim 1-a\left(t / t_{w}\right)^{y}$ proposed in [1]] works also quite well for our data, although not as convincingly as equation 
(6).

Guided by equations (4) and (5) we tried to put our results into the following scaling form:

$$
C\left(t, t_{w}\right)=c_{T} t^{-x(T)} \Phi_{T}\left(t / t_{w}\right)
$$

where $\Phi_{T}(y)=1$ for $y=0$ and $\Phi_{T}(y) \propto y^{x(T)-\tilde{\lambda}(T)}$ for $y \rightarrow \infty$. The form (7) has recently been used 27] successfully to extract the critical dynamical exponent $z$ from the nonequilibrium correlation function (2) via finite size scaling, where the waiting time $t_{w}$ has been replaced by the relaxation time $\tau \propto L^{z}$ in the critical region. For temperatures below $T=0.8$ equation (7) yields an acceptable fit (which can already be deduced from the neglegible waiting time dependence of the exponents $\lambda\left(T, t_{w}\right)$ for $T \leq 0.7$, see fig. 2$)$.

Concluding we have reported new results of numerical nonequilibrium simulations that show an excellent concurrence with experiments on the short range Ising spin glass $\mathrm{Fe}_{0.5} \mathrm{Mn}_{0.5} \mathrm{TiO}_{3}$ and on amorphous metallic spin glasses: not only a single exponent but a whole continuum of (temperature dependent) exponents for the remanent magnetization are found to agree within the numerical errors. Although the values for the exponents extracted from experiments might vary somewhat depending on the microscopic details (range of interactions, spin-type) the main features of the relaxation and the dynamics of many different three-dimensional spin glasses are very similar and the functional forms of the remanent magnetization decay should be the same for different systems [28].

Furthermore we have shown that aging phenomena in the spin glass model under consideration can be observed via the measurement of a particular correlation function and that its nonequilibrium dynamics is indeed gouverned by its equilibrium characteristics for time scales smaller than the imposed waiting (or aging) time. This gives an interesting new perspective (see also [27,29]) to extract equilibrium quantities, which are hard to obtain via MC simulations within the spin glass phase. Finally, by observing plateaus in the short time behavior and slowing down of the algebraic decay of the remanent magnetization, we revealed a dynamical scenario at very low temperatures that is not yet fully understood. 
The author would like to thank A. P. Young for many extremely valuable discussions. He is grateful to J. O. Andersson, D. Belanger, J. P. Bouchaud and P. Nordblad for various comments, hints, suggestions and explanations. The simulations were performed on the CRAY Y-MP at the supercomputer center in Jülich and took about 100 hours of CPU-time. Financial support from the DFG (Deutsche Forschungsgemeinschaft) is also acknowledged. 


\section{REFERENCES}

[1] For a review of spin glasses see K. Binder, and A. P. Young, Rev. Mod. Phys. 58, 801 (1986).

[2] L. Lundgren, R. Svedlindh, P. Nordblad and O. Beckman, Phys. Rev. Lett. 51, 911 (1983).

[3] R. V. Chamberlin, G. Mozurkevich and R. Orbach, Phys. Rev. Lett. 52, 867 (1984);

R. Hoogerbeets, Wei-Li Luo and R. Orbach Phys. Rev. B 34 1719, (1986); M. Alba, J. Hamman, M. Ocio, Ph. Refregier and H. Bouchiat, J. Appl. Phys. 61, 3683 (1987);

M. Ledermann, R. Orbach, J. M. Hammann, M. Ocio and E. Vincent, Phys. Rev. B 44, 7403 (1991).

[4] A. Ito, H. Aruga, E. Torikai, M. Kikuchi, Y. Syono and H. Takai, Phys. Rev. Lett. 57, $483(1986)$.

[5] K. Gunnarson, P. Svedlindh, P. Nordblad, L. Lundgren, H. Aruga and A. Ito, Phys. Rev. Lett. 61, 754 (1988).

[6] P. Granberg, P. Svedlindh, P. Nordblad, L. Lundgren and H. S. Chen, Phys. Rev. B 35, 2075 (1987).

[7] R. G. Palmer, D. L. Stein, E. Abrahams and P. W. Anderson, Phys. Rev. Lett. 53, 958 (1984).

[8] G. J. M. Koper and H. J. Hilhorst, J. Phys. France 49, 429 (1988).

[9] D. S. Fisher and D. A. Huse, Phys. Rev. B 38, 373 (1988).

[10] P. Sibani and K. H. Hoffmann, Phys. Rev. Lett. 63, 2853 (1989).

[11] J. P. Bouchaud, J. Physique I 2, 1705 (1992); J. P. Bouchaud, E. Vincent and J. Hammann, Preprint (1993).

[12] E. Gardner, B. Derrida and P. Mottishaw, J. Phys. France 48, 741 (1987); M. Schreckenberg and H. Rieger, Z. Phys. B 86, 443 (1992).

[13] H. Horner, Z. Phys. B 78, 27 (1990).

[14] H. Eissfeller and M. Opper, Phys. Rev. Lett. 68, 2094 (1992).

[15] H. Sompolinsky and A. Zippelius, Phys. Rev. Lett. 47, 359 (1981). 
[16] R. N. Bhatt and A. P. Young, Phys. Rev. Lett. 54, 924 (1985); Phys. Rev. B 37, 5606 (1988).

[17] A. T. Ogielski and I. Morgenstern, Phys. Rev. Lett. 54, 928 (1985).

[18] A. T. Ogielski, Phys. Rev. B 32, 7384 (1985).

[19] W. Kinzel, Phys. Rev. B 33, 5086 (1986).

[20] D. Huse, Phys. Rev. B 40, 304 (1989).

[21] J. O. Andersson, J. Mattson and P. Svedlindh, Phys. Rev. B 46, 8297 (1992).

[22] G. Parisi and F. Ritort, Preprint ROM2F/92/40.

[23] H. O. Heuer, Comp. Phys. Comm. 59, 387 (1990); H. Rieger, J. Stat. Phys. 70, 1063 (1993).

[24] P. Nordblad, P. Svedlindh, L. Lundgren and L. Sandlund, Phys. Rev. B 33, 645 (1986);

M. Alba, M. Ocio and J. Hammann, Europhys. Lett. 2, 45 (1986).

[25] H. Rieger, unpublished.

[26] According to D. S. Fisher and D. A. Huse, Phys. Rev. Lett. 56, 1601 (1986), also the equilibrium autocorrelation function $q(t)$ should decay logarithmically $q(t) \propto(\ln t)^{-\theta / \psi}$.

[27] R. N. Bhatt and A. P. Young, Europhys. Let. 20, 59 (1992).

[28] P. Nordblad, private communication.

[29] R. E. Blundell, K. Humayun and A. J. Bray, J. Phys. A 25, L733 (1992). 


\section{FIGURES}

FIG. 1. The remanent Magnetization $M_{\text {rem }}(t)$ versus the time $t$ in a log-log plot for varying temperatures. From top to bottom we have $T=0.4,0.5,0.6,0.7,0.8,0.9,1.0$ and 1.1 . The sytem size is $\mathrm{L}=32$ and the data are averaged over 128 samples. The errorbars are of the size of the symbols for $M_{\text {rem }} \leq 0.01$ and much smaller for larger $M_{\text {rem }}$.

FIG. 2. Upper curve: The exponent $\lambda(T)$ for the remanent magnetization (3) versus temperature. The points represented by diamonds $(\diamond$, plus errorbars) are those extracted from figure 11 and the full curve is a least square fit to a quadratic polynomial as a guideline to the eye.

Lower Points: The nonequilibrium exponent $\lambda\left(T, t_{w}\right)$ (see equation (5)) extracted from the long-time behavior $\left(t \gg t_{w}\right)$ of the nonequilibrium correlation function $C\left(t, t_{w}\right)$ (see figure B) for fixed values of $t_{w}$ versus the temperature $T$. From top to bottom we have: $(\triangle) t_{w}=10,(\square)$ $t_{w}=100$ and (o) $t_{w}=1000$. The errorbars are indicated.

FIG. 3. The averaged nonequilibrium spin autocorrelation function $C\left(t, t_{w}\right)$ of equation (2) for fixed values of $t_{w}$ versus time $t$ on a double logarithmic time-scale. The temperature is fixed to be $\mathrm{T}=0.8$ and from top to bottom we have $t_{w}=10^{5}, 10^{4}, 10^{3}, 10^{2}$ and 10 . The system size is $\mathrm{L}=32$ and the data are averaged over 128 samples. The size of the errorbars is only a fraction of the size of the symbols.

FIG. 4. The equilibrium exponent $x(T)$ for the equilibrium autocorrelation funcion $q(t)$ extracted from the short-time behavior $\left(t \ll t_{w}\right)$ of the nonequilibrium correlation function $C\left(t, t_{w}\right)$ (see equation (6) ) versus the temperature $T$. The errorbars are smaller than the circles, as indicated. For $T \leq 0.8$ the data are fitted to a straight line, which shows that at approximately $T=0.3$ the exponent $x(T)$ vanishes. 


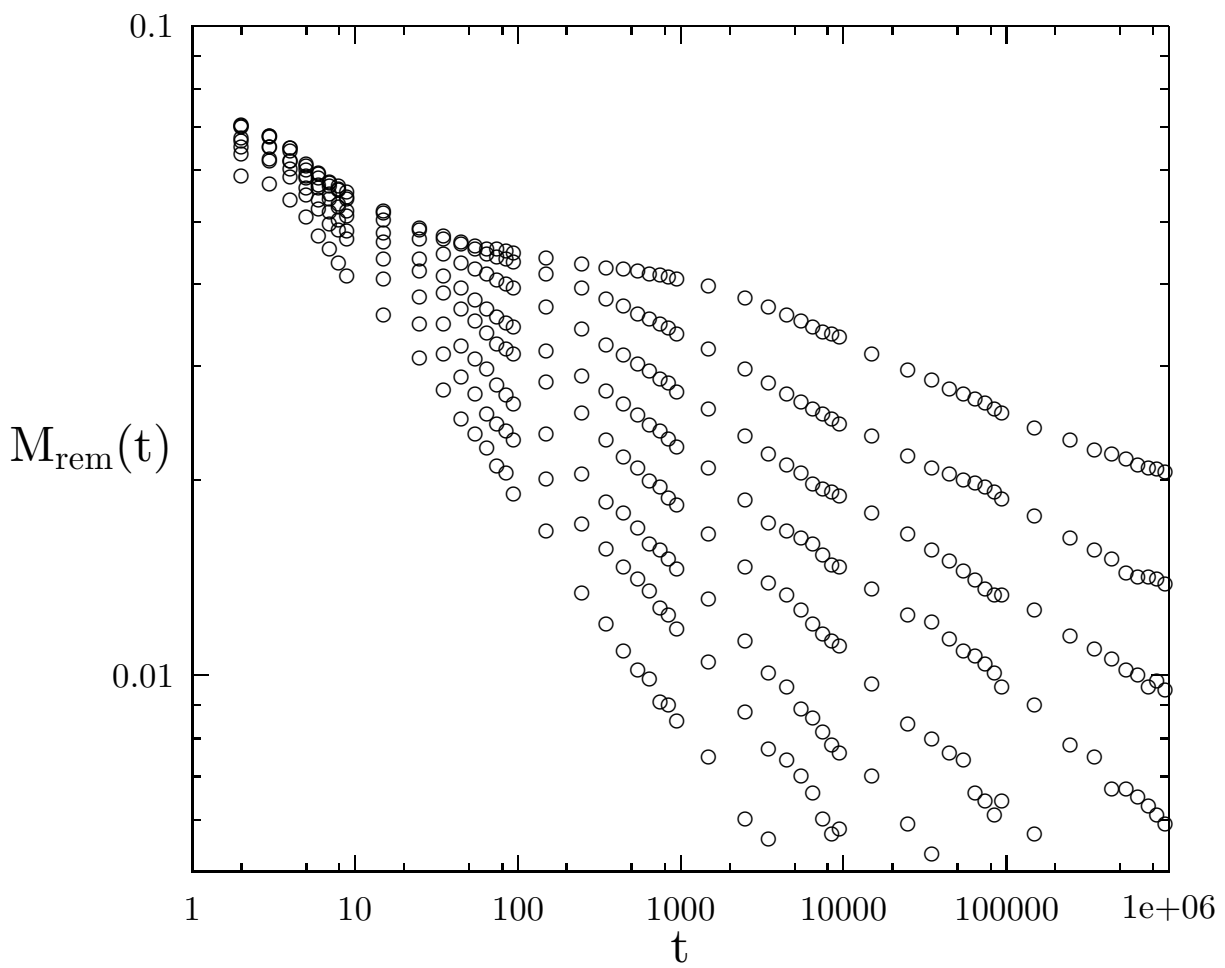

Fig. 1 


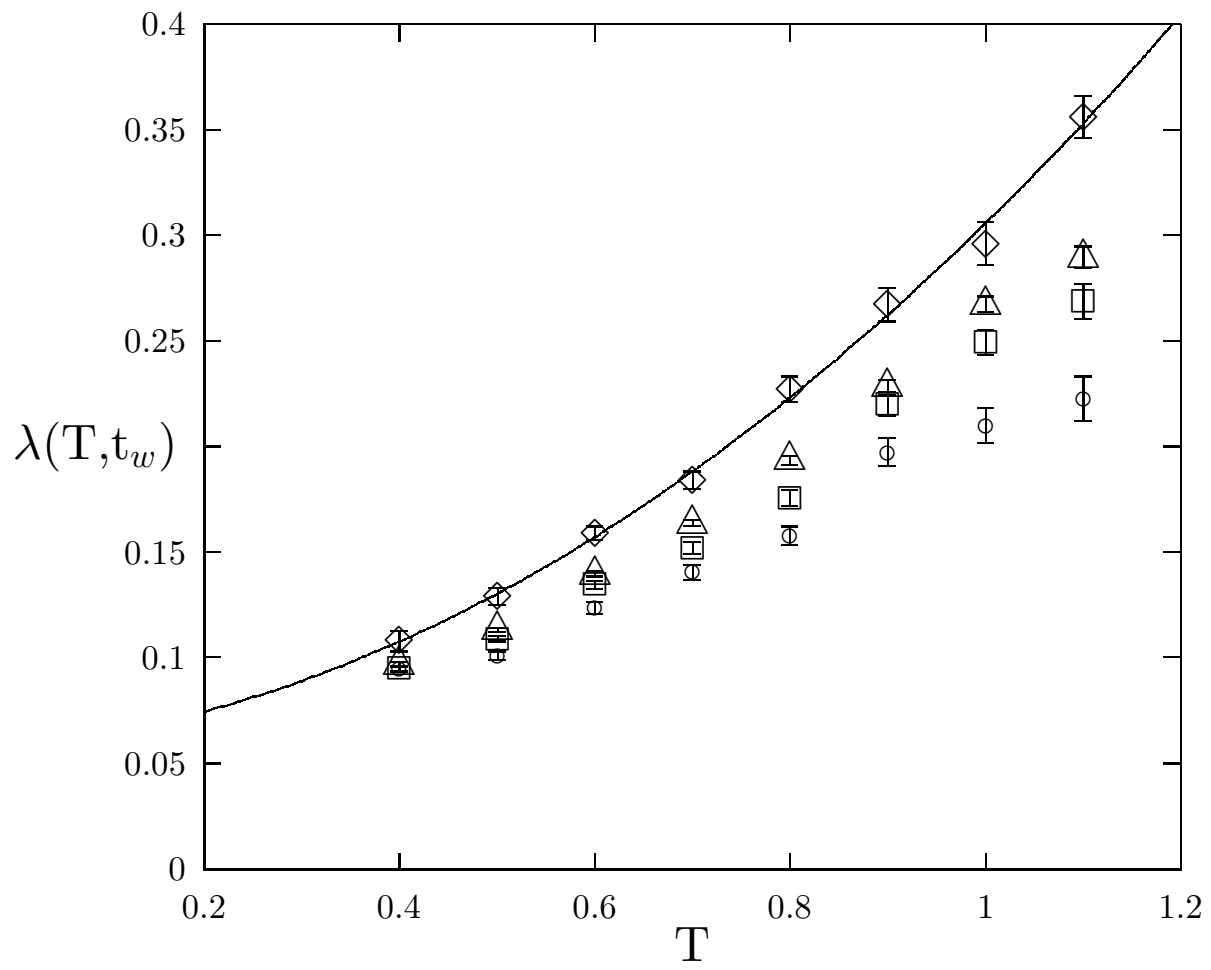

Fig. 2 


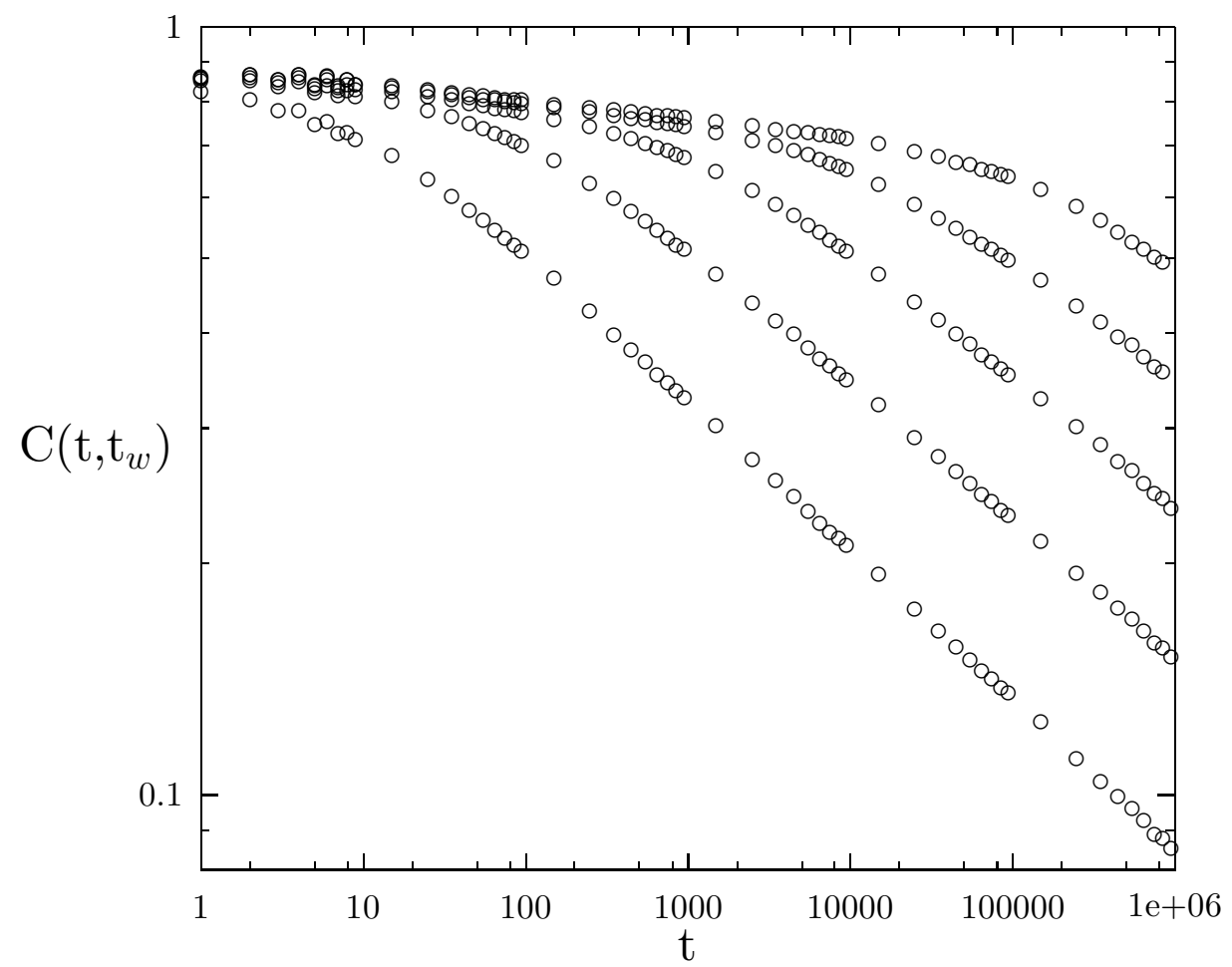

Fig. 3 


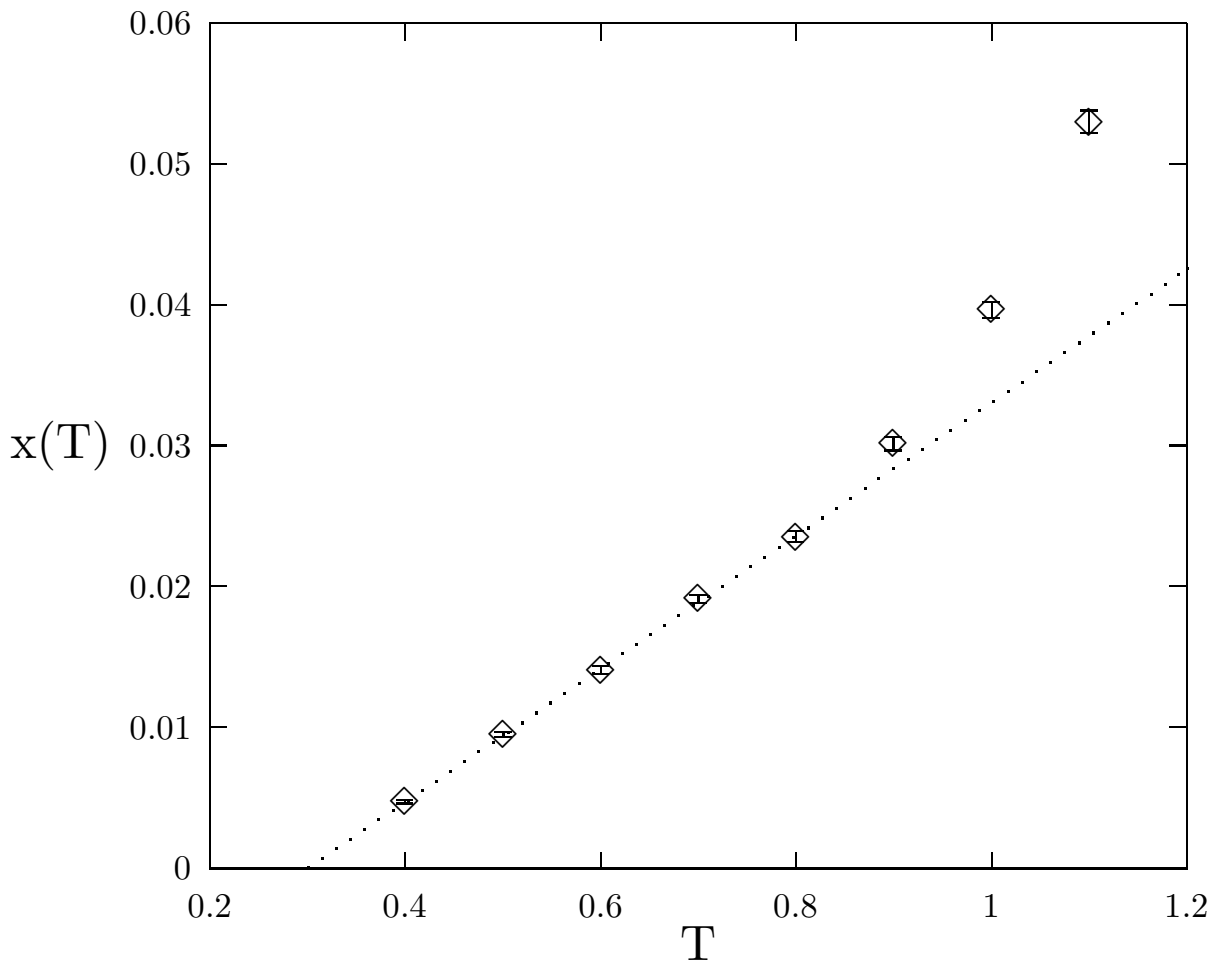

Fig. 4 